\title{
Putative Association of ITGB1 Haplotype with the Clearance of HBV Infection
}

Tae Joon Park', Ji Yong Chun ${ }^{1}$, Joon Seol $\mathrm{Bae}^{1}$, Jason Y. Kim ${ }^{1}$, Jin Sol Lee ${ }^{1}$, Charisse Flerida Pasaje ${ }^{1}$, Byung Lae Park', Hyun Sub Cheong ${ }^{2}$, Hyo-Suk Lee, Yoon Jun $\mathrm{Kim}^{3}$ and Hyoung Doo Shin ${ }^{1,2 *}$

${ }^{1}$ Department of Life Science, Sogang University, Seoul 121-742, Korea, ${ }^{2}$ Department of Genetic Epidemiology, SNP Genetics, Inc., Seoul 153-801, Korea, ${ }^{3}$ Department of Internal Medicine and Liver Research Institute, Seoul National University College of Medicine, Seoul 110-744, Korea

\begin{abstract}
Integrins are transmembrane receptor proteins that mediate cell-cell adhesion and cell-extracellular matrix (ECM) adhesion. The deregulation of cell-ECM adhesion and the abnormal expression of beta1 $(\beta 1)$ integrins (ITGB1S) are involved in tumor development and metastasis. In the liver, the expression of integrins and ECM proteins can be a cause of hepatocellular carcinoma (HCC) development. We performed direct DNA sequencing of 24 individuals, and identified 23 sequence variants of ITGB1 polymorphisms. Among these 23 variants, 7 common variants were selected based on frequencies and linkage disequilibrium, and then genotyped in a larger-scale group of subjects $(n=1,103)$. The genetic associations of ITGB1 polymorphisms with the clearance of HBV and HCC outcome of HBV patients were analyzed using logistic regression models and Cox relative hazard models. Although there was no significant association observed between the polymorphisms and the HCC outcome of HBV patients, the second most common haplotype (ITGB1 haplotype-2 $[C-C-C-C-T-C-T])$ was putatively associated with HBV clearance $\left(\mathrm{OR}=0.75, \mathrm{p}=0.008\right.$ and $\left.P^{\text {corr }}=0.05\right)$. The minor allele frequency (MAF) of ITGB1 haplotype-2 of the spontaneously recovered (SR) group was significantly higher than that of the chronic carrier group (CC) (freq. $=0.248$ vs .0 .199 ). The information derived from this study could be valuable for understanding the genetic factors involved in the clearance of HBV.
\end{abstract}

*Corresponding author: E-mail hdshin@sogang.ac.kr Tel +82-2-705-8615, Fax +82-2-2026-4299 Accepted 26 February 2010
Keywords: Beta integrin ( $\beta$ integrin), Hepatitis $\mathrm{B}$ virus (HBV), Hepatocellular carcinoma (HCC), Liver cirrhosis (LC), Polymorphism

\section{Introduction}

The hepatitis $B$ virus (HBV) is a crucial factor in bringing about acute and chronic liver diseases (Lin et al., 2005). Approximately 350 million people are chronically infected with HBV all over the world. In fact, more than 1 million HBV carriers die annually because of HBV-related diseases such as liver cancer (Chang et al., 2005). Each year, nearly 50 million cases are newly diagnosed, and out of that, $90 \%$ of the affected individuals were infants while the remaining $5 \sim 10 \%$ were adults (Merican et al., 2000). Continuous HBV infection can cause liver cirrhosis (LC) and hepatocellular carcinoma (HCC) (Lin et al., 2005). HCC is the most widespread and severe form of malignancies that are diagnosed in adults. Patients infected with the hepatitis B or C virus are more likely to develop HCC, which in turn is accompanied by liver cirrhosis. The progression of HCC results in a stepwise series of events. Each step in the development of HCC appears to be linked to separate genetic and epigenetic aberrations. These changes are associated with alterations in the expression or formation of an oncogene or a tumor suppressor gene (Patil et al., 2009). In addition, several previous studies have also reported associations between genetic polymorphisms and the risk of HCC and/or HBV clearance, e.g., histone deacetylase-10 (HDAC10) and secreted phosphoprotein-1 (SPP1) polymorphisms, and interleukin-10 (IL19) haplotypes were also shown to be associated with HBV clearance and/or HCC development (Lin et al., 2005; Park et al., 2007; Shin et al., 2003; Shin et al., 2007).

Members of the integrin family, the beta1 $(\beta 1)$ integrins (ITGB1; MIM\# 135630) are heterodimeric structures consisting of a common $\beta 1$ subunit that is non-covalently associated with one of nine different $\alpha$ subunits. These molecules are widely distributed in various cells and they mediate cell-cell and cell-ECM interactions that are related to many biological functions in the development of cell or tissues, hemostasis and immune response (Garrido et al., 2001). It has been shown that the deregulation of cell adhesion to the ECM and the abnormal expression of ITGB1s, particularly the $\alpha 5$ $\beta 1$ down-regulation, are closely associated with tumor development and metastasis. Especially in the liver, the 
expression of integrins and ECM proteins has been linked to HBV infection and HCC development (Lara-Pezzi et al., 2001; Lee et al., 2009).

Based on these observations in the area of tumor and cancer development, we hypothesized that the polymorphisms in the ITGB1 gene could influence the clearance of HBV and HCC progression among HBV-infected patients. We performed extensive screening of ITGB1 by direct sequencing to detect polymorphisms, and we examined their genetic associations with HBV clearance and HCC progression. Here, we report 23 genetic polymorphisms identified in ITGB1 and their genetic associations with HBV clearance and HCC progression in a Korean population $(n=1,103)$.

\section{Methods}

\section{Subjects and outcomes}

A total of 1,103 Korean subjects having either present or past evidence of HBV infection were prospectively extracted from the outpatient clinic of the liver unit and from the Center for Health Promotion of Seoul National University Hospital from January 2001 to August 2003. These subjects were divided into two groups according to their serologic markers: the chronic carrier (CC) group and the spontaneously recovered (SR) group. The CC and SR groups were composed of 670 and 433 subjects, respectively (Table 1). The HBsAg-positive patients (CC group) were hepatitis B surface antigen (HBsAg)positive over a 6-month period. They were followed up for disease progression at least every 6 months. The diagnoses of the CC and SR subjects were established by repeated seropositivity of the hepatitis $B$ surface antigen (Enzygnost ${ }^{R}$ HBsAg 5.0; Dade Behring, Marburg,

Table 1. Clinical profiles of study subjects

\begin{tabular}{lccc}
\hline & & \multicolumn{2}{c}{$\mathrm{CC}$} \\
\cline { 3 - 4 } & & $\mathrm{CH}$ or LC & HCC \\
\hline No. of subjects & 433 & 343 & 327 \\
Age (mean (range)) & 54.9 & 49.8 & 58.3 \\
& $(28 \sim 79)$ & $(22 \sim 85)$ & $(25 \sim 79)$ \\
Sex (male/female) & $240 / 193$ & $278 / 65$ & $279 / 48$ \\
HBeAg (positive rate, \%) & 0 & 33.2 & 19.6 \\
HBeAb (positive rate, \%) & 0 & 30 & 43.4 \\
HBsAg (positive rate, \%) & 0 & 100 & 100 \\
HBsAb (positive rate, \%) & 100 & 0 & 0.3 \\
U albumin (positive rate, \%) & 0 & 7 & 13.5 \\
U blood (positive rate, \%) & 28.2 & 12 & 22.9 \\
\hline
\end{tabular}

$\mathrm{SR}$, spontaneously recovered; $\mathrm{CH}$, chronic hepatitis; $\mathrm{CC}$, chronic carrier; LC, liver cirrhosis; HCC, hepatocellular carcinoma; U, Urine.
Germany), anti-HBs (Enzygnost ${ }^{\circledR}$ Anti-HBs II) and anti-HBc (AB-Corek; DiaSorin s.r.I., Saluggia, Italy) of the IgG type without $\mathrm{HBsAg}$, respectively, over a 6-month period. The CC group was further divided into two subgroups, i.e., those without (the $\mathrm{CH} / \mathrm{LC}$ group; $\mathrm{n}=343$ ) and those with HCC (the HCC group; $n=327$ ), according to the absence or presence of HCC, respectively. We excluded subjects who were positive for anti-HBs only and not for anti-HBc, as well as those who were positive for anti-HCV and anti-HIV (GENEDIA ${ }^{\mathrm{R}}$; Greencross Life Science Corp., Yongin, Korea, $\mathrm{HCV}^{\mathbb{R}} 3.2$; Dong-A Pharmaceutical Co., Seoul, Korea). The patients who had other types of liver disease such as autoimmune hepatitis, toxic hepatitis, primary biliary cirrhosis, or Budd-Chiari syndrome were also excluded from the sample. None of the patients had a previous history of immunosuppression or anti-viral treatment.

Informed consent was gained from each patient, and the Institutional Review Board of Human Research at Seoul National University Hospital approved the study protocol. Liver cirrhosis was diagnosed pathologically or by the clinical evidences of portal hypertension such as visible collateral vessels on the abdominal wall, esophageal varices on the esophagogastroscopy, palpable splenomegaly, and sonographically definite findings of cirrhotic liver or ascites. HCC was diagnosed as described previously (Bruix et al., 2001). The clinical parameters are summarized in Table 1.

\section{Sequencing analysis of ITGB1}

Using the $\mathrm{ABI}$ PRISM 3730 DNA analyzer (Applied Biosystems, Foster City, CA), we sequenced all exons, including exon-intron boundaries and promoter regions $(\sim 1.5 \mathrm{~kb})$, to discover polymorphisms of the /TGB1 gene using DNA samples of 24 unrelated healthy Korean individuals. Twenty seven primer sets for amplification (Supplementary Table 1) and sequencing analysis were designed based on GenBank sequences (NT_008705.15). Sequence variants were verified by chromatograms.

\section{Genotyping with fluorescence polarization de- tection}

In order to genotype the polymorphic sites in our study, amplifying primers and probes were designed for TaqMan (Livak, 1999). Primer Express (Applied Biosystems) was used to design both the PCR primers and the MGB TaqMan probes. One allelic probe was labeled with the FAM dye and the other with the fluorescent VIC dye (Supplementary Table 2). Typically, PCR was run in the TaqMan Universal Master mix without UNG (Applied Biosystems) at a primer concentration of $900 \mathrm{nM}$ and a 
TaqMan MGB-probe concentration of $200 \mathrm{nM}$. The reaction was performed in a 384-well format in a total reaction volume of 5 ul using $20 \mathrm{ng}$ of genomic DNA. The plate was then placed in a thermal cycler (PE 9700, Applied Biosystems) and heated for $2 \mathrm{~min}$ at $50^{\circ} \mathrm{C}$ and for $10 \mathrm{~min}$ at $95^{\circ} \mathrm{C}$, followed by 40 cycles of $95^{\circ} \mathrm{C}$ for $15 \mathrm{~s}$ and $60^{\circ} \mathrm{C}$ for $1 \mathrm{~min}$. The TaqMan assay plate was then transferred to a Prism $7900 \mathrm{HT}$ instrument (Applied Biosystems) where the fluorescence intensity of each well was read. Fluorescence data files from each plate were analyzed by automated software (SDS 2.1). Primer sequences are listed in Supplementary Table 1.

\section{Statistical analysis}

Linkage disequilibrium (LD) was inferred using the algorithm (Haploview) that searches for a spine of strong ID' I and LD coefficient $r^{2}$ running from one marker to another (Barrett et al., 2005). Haplotypes of each individual were determined using the algorithm (PHASE, version 2.0) developed by Stephens et al (Stephens et al., 2001). Subjects with missing genotypes were omit- ted in the analysis of individual single-nucleotide polymorphisms (SNPs) and haplotypes. The genotyping success rate was $>99 \%$, which makes the omission of a few individuals unlikely to change the results of the analysis. For analysis of viral clearance as an outcome, logistic regression models were used for calculating odds ratios (95\% confidential interval) and corresponding $p$-values controlling for age (continuous value) and sex (male $=0$, female $=1$ ) as covariates. Cox models were used for calculating relative hazards and P-values controlling for sex and status of liver cirrhosis among the CC group.

The effective number of independent marker loci in ITGB1 was calculated to correct for multiple testing, using the software SNPSpD (http://genepi.qimr.edu.au/ general/daleN/SNPSpD/), which is based on spectral decomposition $(\mathrm{SpD})$ of matrices of pair-wise LD between SNPs (Nyholt, 2004). The resulting number of independent marker loci was applied to check for multiple testing. Statistical powers were calculated using the software, "Power for Genetic Association Analyses" (PGA) (Menashe et al., 2008). PGA is an application

\section{A. Map of ITGB1 (integrin, beta 1) on 10p11.2 (59.5 kb) isoform (mRNA:NM_002211)}
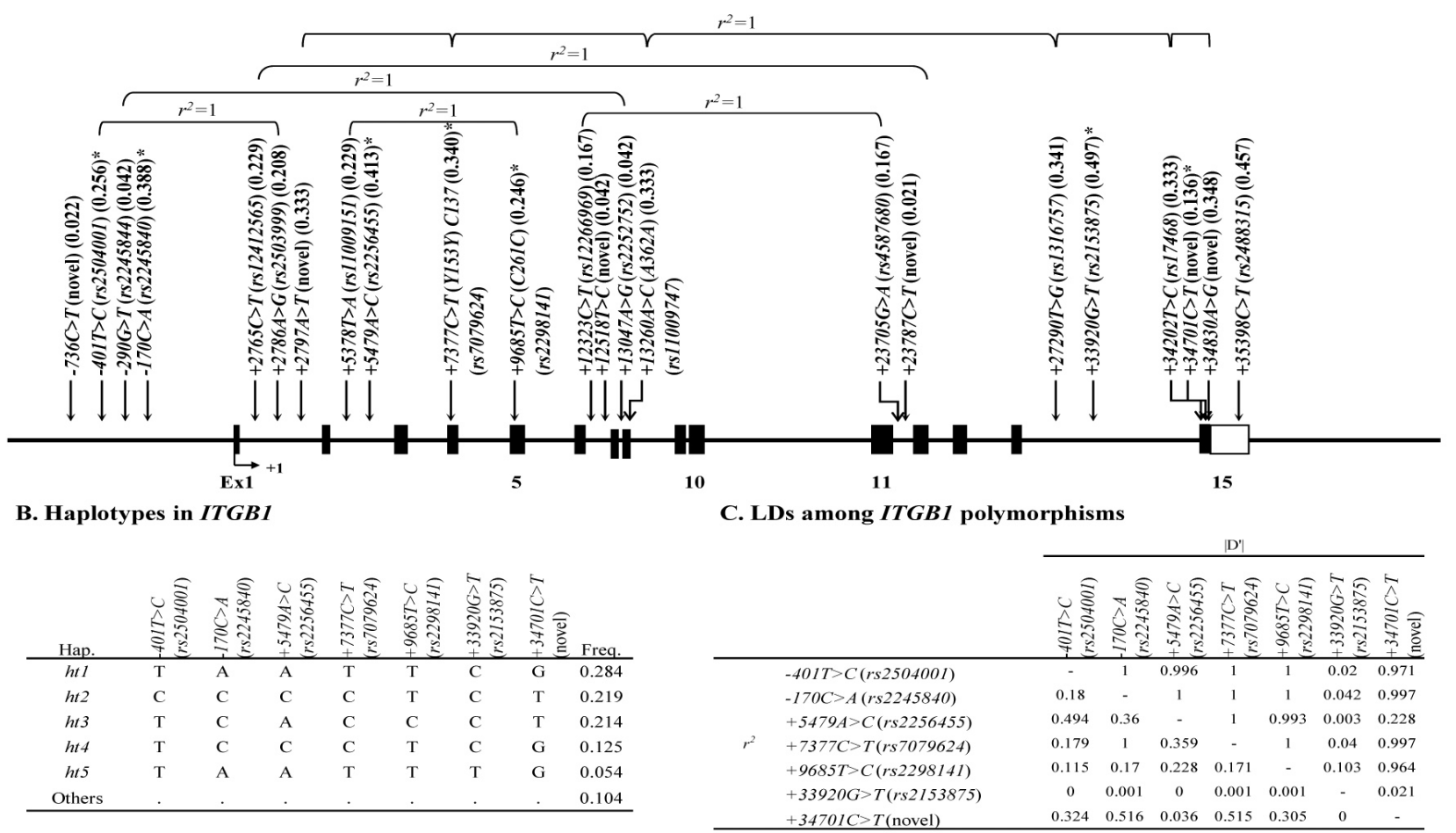

Fig. 1. Gene maps and haplotypes of the ITGB1 gene. A. Polymorphisms identified in ITGB1. Coding exons are marked by shaded blocks and 3'-UTR by white blocks. Asterisks $\left(^{*}\right)$ indicate SNPs that were genotyped in the larger population. The LD coefficients $\left(r^{2}\right)$ are based on the genotypes of Korean samples. B. Haplotypes of ITGB1 in the Korean population. Only those with frequencies over 0.05 are shown. C. LD coefficients (ID'l and $r^{2}$ ) among the selected SNPs based on the genotypes of whole study subjects in this study $(n=1,103)$. 
specifically designed to calculate statistical power and other values in case-control association studies. A co-dominant (1df) model with relative risk 1.3, disease prevalence value of HBV $7.1 \%$ (Lee et al., 1998), effective degree of freedom (EDF) 2, and an alpha error level of $5 \%$ was used to calculate the statistical power.

\section{Results}

Through direct sequencing of 24 individuals, we identified 23 sequence variants in the ITGB1 gene: 4 in the promoter region, 7 in coding regions of exons, 11 in introns, and 1 in the 3 '-untranslated region (3'-UTR) (Fig. $1 \mathrm{~A})$. Pairwise comparisons of all 23 polymorphisms revealed two sets of markers in absolute linkage disequilibrium (LD) ( $\left|D^{\prime}\right|=1$ and $r^{2}=1$, Fig. 1A). Among these 23 variants, 7 common polymorphisms $(-401 T>$ $C,-170 C>A,+5479 A>C,+7377 C>T,+9685 T>$ $C$, $+33920 G>T$, and $+34701 C>T$ ) were selected for larger-scale genotyping $(n=1,103)$ based on location, minor allele frequency (MAF>0.05), and LD. No significant deviations from Hardy-Weinberg equilibrium (HWE) were observed ( $p>0.05$, Table 2). Five major haplotypes showed frequencies greater than 0.05 and accounted for over $89.6 \%$ of the distribution (Fig. 1B). Statistical powers of each polymorphism are shown in Table 3.

Association analyses of HBV clearance (CC vs. SR) and HCC occurrence (HCC vs. CH/LC) for each polymorphism and haplotype of the ITGB1 gene were performed using logistic regression models, controlling for age and sex as covariates (Table 3). The P-values and odds ratios of each polymorphism and haplotype are shown in Table 3. Among polymorphisms, the second most common haplotype of the ITGB1 gene, ITGB1 haplotype-2 $[C-C-C-C-T-C-T]$, was found to be putatively associated with HBV clearance, i.e., the frequency of individuals bearing the ITGB1 haplotype-2 allele among the SR group was significantly higher than those in the CC group (freq. $=0.248$ vs. 0.199, OR=0.75, $\left.\mathrm{p}=0.008, P^{\text {corr }}=0.05\right)$. Although the significances were not retained after correction for multiple testing, similar associations were also observed in $-401 T>C$ (rs2504001) and +33920G $>T($ rs2153875). There was no association observed between ITGB1 polymorphisms or haplotypes and HCC occurrence (Table 3).

To analyze the role of ITGB1 polymorphisms in the onset age of $\mathrm{HCC}$, Cox relative hazards analysis for age of HCC occurrence was performed for the CC group. No significant association was observed (Table 3).

\section{Discussion}

Integrins play a major role in cell-cell and cell-ECM adhesion. They mediate signaling cascades that are involved in many cell functions. Up-regulated $\beta$ integrins in the vascular endothelium function as angiogenesis inhibitors in blood vessels of some human tumors (Bridger et al., 2008; Carlson et al., 2008). The abnormal expression of $\beta$ integrins and the deregulation of cellECM adhesion can be causes of tumor cell development, growth, and metastasis. Previous studies have suggested that the down-regulation of integrin $\alpha 5 \beta 1$ is associated with the growth of tumor cells (Lara-Pezzi et al., 2001; Bridger et al., 2008), and higher expression of $\beta 1$ integrin (/TGB1) mRNA is significantly associated with docetaxel resistance, which inhibits the effect of chemotherapy for esophageal squamous cell carcinoma (Mori et al., 2008). In addition, $\beta$ integrins have key roles in the primary tumor formation, metastatic dissemination and inhibition of tumor cell senescence in mouse models which have breast and pancreatic cancer (Streuli and Akhtar, 2009).

In this study, we demonstrated that the second most common haplotype, ITGB1 haplotype-2 [C-C-C-C-T$C-T]$, was putatively associated with HBV clearance. In addition, there were association signals in $-401 T>C$ (rs2504001) located in the promoter region and $+33920 G>$ $T$ (rs2153875) in intron 14, although significances were not retained after correction for multiple testing. If the promoter polymorphism is located in transcription binding sites, it can induce the alteration of the transcription factor binding and affects transcriptional regulation. Previous studies demonstrated the associations of SNPs in promoter regions with $\mathrm{HBV}$ infection and/or HCC occurrence. First of all, histone deacetylase 10 (HDAC10) polymorphism (HDAC10-589C)T) was associated with the clearance of HBV infection and onset age of HCC. The promoter activity of this SNP was measured by luciferase activity reporter assay. The functional assay showed that luciferase activity of "T" allele was significantly higher than that of "C" allele of HDAC10-589C > $\mathrm{T}$ (Park et al., 2007). Second, interleukin-18 (/L-18) polymorphism $(/ L-18-148 G>C)$ in promoter is associated with the risk of HCC. The promoter activity of $I L-18-148 G>C$ measured by luciferase assay revealed that $-148 \mathrm{C}$ allele represses transcriptional activity compared with the $-148 G$ allele (Kim et al., 2009). In addition, the promoter SNPs of tumor necrosis factor- $\alpha$ $(T N F-\alpha)$ (TNF- $\alpha-863$ and TNF- $\alpha-308$ ) (Kim et al., 2003b) and transforming growth factor- $\beta 1$ (TGF- $\beta 1$ ) (TGF- $\beta$ 1-509) were associated with HBV clearance and/or HCC occurrence (Kim et al., 2003a).

The involvements of $\beta$ integrin polymorphisms in HBV infection and HCC development have not been reported in previous studies. However, SNPs in other family member of integrin genes showed associations with 
Table 2. Genotype and allele frequency of polymorphisms in ITGB1

\begin{tabular}{|c|c|c|c|c|c|c|c|c|c|c|}
\hline \multirow{2}{*}{$\begin{array}{c}\text { Loci } \\
-736 C>T\end{array}$} & \multirow{2}{*}{$\begin{array}{c}\text { Position } \\
\text { Promoter }\end{array}$} & \multirow{2}{*}{$\begin{array}{c}\text { Amino acid } \\
\text { change }\end{array}$} & \multicolumn{2}{|l|}{ rs\# } & \multicolumn{3}{|c|}{ Genotype } & \multirow{2}{*}{$\begin{array}{c}\text { Frequency } \\
0.022\end{array}$} & \multirow{2}{*}{$\begin{array}{c}\text { Heterozygosity } \\
0.043\end{array}$} & \multirow{2}{*}{$\begin{array}{l}\text { HWE } \\
0.915\end{array}$} \\
\hline & & & Novel & C & $\mathrm{CT}$ & T & $N$ & & & \\
\hline & & & & 22 & 1 & 0 & 23 & & & \\
\hline \multirow{2}{*}{$-401 T>C$} & Promoter & - & rs2504001 & $\mathrm{T}$ & CT & C & $\mathrm{N}$ & 0.256 & 0.381 & 0.290 \\
\hline & & & & 608 & 401 & 78 & 1087 & & & \\
\hline \multirow[t]{2}{*}{$-290 G>T$} & Promoter & & rs2245844 & $\mathrm{G}$ & GT & $T$ & $\mathrm{~N}$ & 0.042 & 0.080 & 0.831 \\
\hline & & & & 22 & 2 & 0 & 24 & & & \\
\hline \multirow[t]{2}{*}{$-170 C>A$} & Promoter & - & rs2245840 & $C$ & $A C$ & $A$ & $\mathrm{~N}$ & 0.338 & 0.447 & 0.962 \\
\hline & & & & 474 & 487 & 123 & 1084 & & & \\
\hline \multirow[t]{2}{*}{$+2765 C>T$} & Intron1 & & rs12412565 & $C$ & $\mathrm{CT}$ & $\mathrm{T}$ & $\mathrm{N}$ & 0.229 & 0.353 & 0.763 \\
\hline & & & & 14 & 9 & 1 & 24 & & & \\
\hline \multirow[t]{2}{*}{$+2786 A>G$} & Intron1 & & rs2503999 & $A$ & $A G$ & G & $\mathrm{N}$ & 0.208 & 0.330 & 0.236 \\
\hline & & & & 16 & 6 & 2 & 24 & & & \\
\hline \multirow[t]{2}{*}{$+2797 A>T$} & Intron1 & & Novel & $A$ & AT & $\mathrm{T}$ & $\mathrm{N}$ & 0.333 & 0.444 & 0.540 \\
\hline & & & & 10 & 12 & 2 & 24 & & & \\
\hline \multirow[t]{2}{*}{$+5378 T>A$} & Intron2 & & rs11009151 & $T$ & AT & A & $\mathrm{N}$ & 0.229 & 0.353 & 0.763 \\
\hline & & & & 14 & 9 & 1 & 24 & & & \\
\hline \multirow[t]{2}{*}{$+5479 A>C$} & Intron2 & - & rs2256455 & $A$ & $A C$ & $C$ & $\mathrm{~N}$ & 0.413 & 0.485 & 0.140 \\
\hline & & & & 371 & 482 & 189 & 1042 & & & \\
\hline \multirow[t]{2}{*}{$+7377 C>T$} & Exon4 & Tyr153Tyr & rs7079624 & $C$ & $\mathrm{CT}$ & $T$ & $\mathrm{~N}$ & 0.340 & 0.449 & 0.828 \\
\hline & & & & 470 & 490 & 124 & 1084 & & & \\
\hline \multirow[t]{2}{*}{$+9685 T>C$} & Exon5 & Cys261Cys & rs2298141 & $\mathrm{T}$ & $\mathrm{CT}$ & $C$ & $N$ & 0.246 & 0.371 & 0.369 \\
\hline & & & & 622 & 391 & 71 & 1084 & & & \\
\hline \multirow[t]{2}{*}{$+12323 C>T$} & Intron6 & & rs12266969 & $C$ & $\mathrm{CT}$ & $T$ & $\mathrm{~N}$ & 0.167 & 0.278 & 0.624 \\
\hline & & & & 17 & 6 & 1 & 24 & & & \\
\hline \multirow[t]{2}{*}{$+12518 T>C$} & Intron6 & & Novel & $T$ & $\mathrm{CT}$ & C & $N$ & 0.042 & 0.080 & 0.000 \\
\hline & & & & 23 & 0 & 1 & 24 & & & \\
\hline$+13047 A>G$ & Intron7 & & rs2252752 & A & $A G$ & $\mathrm{G}$ & $\mathrm{N}$ & 0.042 & 0.080 & 0.831 \\
\hline & & & & 22 & 2 & 0 & 24 & & & \\
\hline$+13260 A>C$ & Exon8 & Ala362Ala & rs 11009147 & $A$ & $A C$ & C & $\mathrm{N}$ & 0.333 & 0.444 & 0.540 \\
\hline & & & & 10 & 12 & 2 & 24 & & & \\
\hline$+23705 G>A$ & Intron11 & & rs4587680 & $G$ & $A G$ & A & $N$ & 0.167 & 0.278 & 0.624 \\
\hline & & & & 17 & 6 & 1 & 24 & & & \\
\hline$+23787 C>T$ & Intron11 & & Novel & $\mathrm{C}$ & $\mathrm{CT}$ & $T$ & $\mathrm{~N}$ & 0.021 & 0.041 & 0.917 \\
\hline & & & & 23 & 1 & 0 & 24 & & & \\
\hline$+27290 T>G$ & Intron14 & & rs1316757 & $\mathrm{T}$ & GT & G & $\mathrm{N}$ & 0.341 & 0.449 & 0.597 \\
\hline & & & & 9 & 11 & 2 & 22 & & & \\
\hline$+33920 G>T$ & Intron14 & - & rs2153875 & $G$ & GT & $T$ & $\mathrm{~N}$ & 0.498 & 0.503 & 0.540 \\
\hline & & & & 273 & 521 & 268 & 1062 & & & \\
\hline$+34202 T>C$ & Exon15 & & rs17468 & $\mathrm{T}$ & $\mathrm{CT}$ & $C$ & $\mathrm{~N}$ & 0.333 & 0.444 & 0.540 \\
\hline & & & & 10 & 12 & 2 & 24 & & & \\
\hline$+34701 C>T$ & Exon15 & - & Novel & $C$ & CT & $T$ & $\mathrm{~N}$ & 0.136 & 0.235 & 0.066 \\
\hline & & & & 805 & 270 & 13 & 1088 & & & \\
\hline$+34830 A>G$ & Exon15 & & Novel & A & $A G$ & $G$ & $\mathrm{~N}$ & 0.348 & 0.454 & 0.472 \\
\hline & & & & 9 & 12 & 2 & 23 & & & \\
\hline$+35398 C>T$ & 3'-UTR & & rs2488315 & C & $\mathrm{CT}$ & $T$ & $\mathrm{~N}$ & 0.457 & 0.496 & 0.311 \\
\hline & & & & 8 & 9 & 6 & 23 & & & \\
\hline
\end{tabular}

Bold-face indicates SNPs selected for larger-scale genotyping.

HBV and HCC. Integrin $\alpha \mathrm{v}$ (ITGAV) SNPs, which are located in introns (rs9333289, rs11685758, and rs2290083) and 3'-UTR (rs1839123), and a haplotype (haplotype-1 $[C-C-G]$ ) were found to be significantly associated with chronic hepatitis and HBV-infected HCC in a Korean population (Lee et al., 2009). In that study, rs9333289, rs11685758 and rs1839123 were associated with susceptibility to HBV-infected HCC, and rs2290083 was associated with susceptibility to both chronic infection of HBV and HBV-infected HCC. In addition, the major hap- 


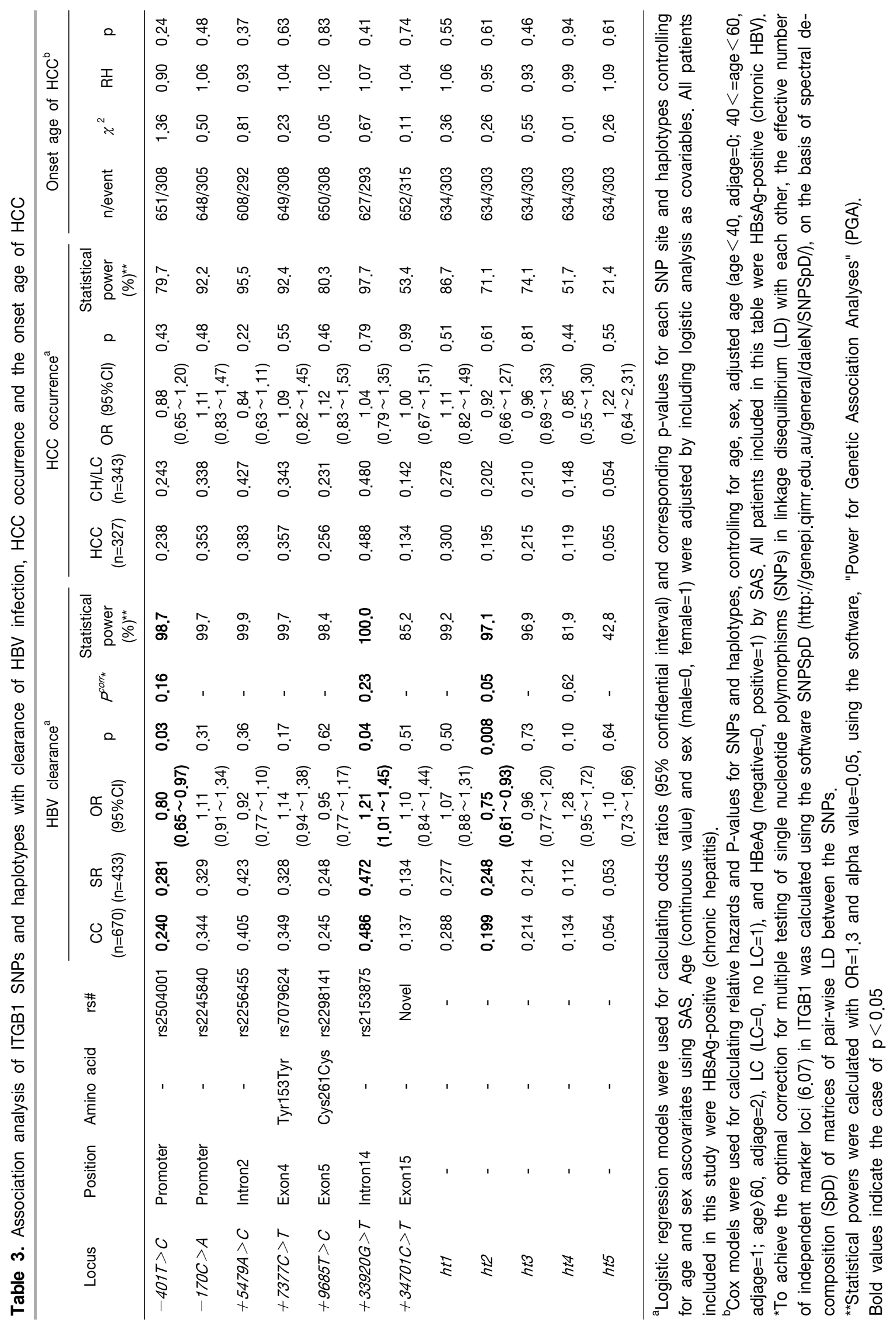


lotype, haplotype-1 [C-C-G] for rs11685758-rs2290083rs1839123, was associated with decreased susceptibility to chronic infection of $\mathrm{HBV}(\mathrm{OR}=0.59,95 \% \mathrm{Cl}=0.36$ $0.97, \mathrm{p}=0.04)$ and HBV-infected HCC (OR=0.58, 95\% $\mathrm{Cl}=0.34 \sim 0.98, \mathrm{p}=0.04)$. In comparison with our study, the association between haplotype-1 $[C-C-G]$ and the decreased susceptibility to chronic HBV infection was similar to our results with ITGB1 haplotype-2 [C-C-C-C$T-C-T]$. The previous study may be less reliable than our study because of relatively small subjects $(n=304)$ and p-values which did not undergo multiple testing corrections. However, in spite of the relatively less reliability, this study demonstrated the association between ITGAV polymorphisms and susceptibility to HBV infection (Lee et al., 2009). Therefore, the alpha subunit of integrin, ITGAV may be a candidate partner for the beta subunit of integrin, ITGB1 in the case of HBV infection.

Additional studies have suggested the associations between integrin polymorphisms in introns and human diseases. In particular, $\alpha 4$ and $\alpha 9$ integrin polymorphisms (ITGA4 and ITGA9, respectively) were shown to be associated with autism and nasopharyngeal carcinoma (NPC), respectively. One ITGA4 polymorphism in intron ( $r$ 155100) showed association with autism in a Portuguese population, and eight ITGA9 intron polymorphisms (rs169188, rs197721, rs149816, rs169111, rs197770, rs2212020, rs189897, and rs197757) were associated with NPC in a Malaysian Chinese population (Correia et al., 2009; Ng et al., 2009). Similar associations between integrin polymorphisms and human diseases in various European populations were proposed by another study, i.e., $\alpha 4$ integrin polymorphisms (ITGA4) (rs1449263 in the promoter and rs3770138 in an intron) were significantly associated with multiple sclerosis (MS) in Basque and Nordic populations (The rs1449263 polymorphism was associated with MS only in the Basque population, whereas the rs3770138 was associated only in the Nordic population.) (O'Doherty et al., 2007).

In summary, we identified 23 genetic variants in the human ITGB1 gene. Seven common polymorphic sites were selected for genotyping in our HBV cohort, and statistical analyses showed that ITGB1 haplotype-2 $[C-C-C-C-T-C-T]$ was putatively associated with HBV clearance. Our findings will provide useful information for further genetic studies of this important gene.

\section{Acknowledgements}

We greatly acknowledge and thank the participants of the study and their families who took part in the HBV cohort study by Seoul National University. This work was supported by a grant from the Korea Science and
Engineering Foundation (KOSEF) funded by the Korean government (MEST) (No. 2009-0080157) as well as the National R\&D Program for Cancer Control, Ministry for Health, Welfare and Family affairs, Republic of Korea (0920280).

\section{References}

Barrett, J.C., Fry, B., Maller, J., and Daly, M.J. (2005). Haploview: analysis and visualization of LD and haplotype maps. Bioinformatics 21, 263-265.

Bridger, P.S., Haupt, S., Leiser, R., Johnson, G.A., Burghardt, R.C., Tinneberg, H.R., and Pfarrer, C. (2008). Integrin activation in bovine placentomes and in caruncular epithelial cells isolated from pregnant cows. Biol. Reprod. 79, 274-282.

Bruix, J., Sherman, M., Llovet, J.M., Beaugrand, M., Lencioni, R., Burroughs, A.K., Christensen, E., Pagliaro, L., Colombo, M., and Rodes, J. (2001). Clinical management of hepatocellular carcinoma. Conclusions of the Barcelona-2000 EASL conference. European Association for the Study of the Liver. J. Hepatol. 35, 421-430.

Carlson, T.R., Hu, H., Braren, R., Kim, Y.H., and Wang, R.A. (2008). Cell-autonomous requirement for beta1 integrin in endothelial cell adhesion, migration and survival during angiogenesis in mice. Development 135, 21932202.

Chang, J.J., Wightman, F., Bartholomeusz, A., Ayres, A., Kent, S.J., Sasadeusz, J., and Lewin, S.R. (2005). Reduced hepatitis B virus (HBV)-specific CD4+ T-cell responses in human immunodeficiency virus type 1-HBV-coinfected individuals receiving HBV-active antiretroviral therapy. J. Virol. 79, 3038-3051.

Correia, C., Coutinho, A.M., Almeida, J., Lontro, R., Lobo, C., Miguel, T.S., Martins, M., Gallagher, L., Conroy, J., Gill, M., Oliveira, G., and Vicente, A.M. (2009). Association of the alpha4 integrin subunit gene (ITGA4) with autism. Am. J. Med. Genet. B. Neuropsychiatr. Genet. 150B, 1147-1151.

Garrido, J.J., Jimenez-Marin, A.M., Yerle, M., de AndresCara, D.F., Morera, L., Llanes, D., and Barbancho, M.J. (2001). Assignment of the ITGB1 (integrin beta1 subunit) gene to pig chromosome band $10 \mathrm{q} 17$ with somatic cell hybrids. Cytogenet. Cell Genet. 94, 84-85.

Kim, Y.J., Lee, H.S., Im, J.P., Min, B.H., Kim, H.D., Jeong, J.B., Yoon, J.H., Kim, C.Y., Kim, M.S., Kim, J.Y., Jung, J.H., Kim, L.H., Park, B.L., and Shin, H.D. (2003a). Association of transforming growth factor-beta1 gene polymorphisms with a hepatocellular carcinoma risk in patients with chronic hepatitis B virus infection. Exp. Mol. Med. 35, 196-202.

Kim, Y.J., Lee, H.S., Yoon, J.H., Kim, C.Y., Park, M.H., Kim, L.H., Park, B.L., and Shin, H.D. (2003b). Association of TNF-alpha promoter polymorphisms with the clearance of hepatitis B virus infection. Hum. Mol. Genet. 12, 25412546

Kim, Y.S., Cheong, J.Y., Cho, S.W., Lee, K.M., Hwang, J.C., Oh, B., Kimm, K., Lee, J.A., Park, B.L., Cheong, 
H.S., Shin, H.D., and Kim, J.H. (2009). A functional SNP of the interleukin-18 gene is associated with the presence of hepatocellular carcinoma in hepatitis B virus-infected patients. Dig. Dis. Sci. 54, 2722-2728.

Lara-Pezzi, E., Majano, P.L., Yanez-Mo, M., GomezGonzalo, M., Carretero, M., Moreno-Otero, R., SanchezMadrid, F., and Lopez-Cabrera, M. (2001). Effect of the hepatitis $B$ virus $\mathrm{HBx}$ protein on integrin-mediated adhesion to and migration on extracellular matrix. J. Hepatol. 34, 409-415.

Lee, M.S., Kim, D.H., Kim, H., Lee, H.S., Kim, C.Y., Park, T.S., Yoo, K.Y., Park, B.J., and Ahn, Y.O. (1998). Hepatitis $B$ vaccination and reduced risk of primary liver cancer among male adults: a cohort study in Korea. Int. J. Epidemiol. 27, 316-319.

Lee, S.K., Kim, M.H., Cheong, J.Y., Cho, S.W., Yang, S.J., and Kwack, K. (2009). Integrin alpha $V$ polymorphisms and haplotypes in a Korean population are associated with susceptibility to chronic hepatitis and hepatocellular carcinoma. Liver Int. 29, 187-195.

Lin, C.L., Liao, L.Y., Wang, C.S., Chen, P.J., Lai, M.Y., Chen, D.S., and Kao, J.H. (2005). Basal core-promoter mutant of hepatitis $B$ virus and progression of liver disease in hepatitis $B$ e antigen-negative chronic hepatitis B. Liver Int. 25, 564-570.

Livak, K.J. (1999). Allelic discrimination using fluorogenic probes and the $5^{\prime}$ nuclease assay. Genet. Anal. 14, 143-149.

Menashe, I., Rosenberg, P.S., and Chen, B.E. (2008). PGA: power calculator for case-control genetic association analyses. BMC Genet. 9, 36.

Merican, I., Guan, R., Amarapuka, D., Alexander, M.J., Chutaputti, A., Chien, R.N., Hasnian, S.S., Leung, N., Lesmana, L., Phiet, P.H., Sjalfoellah Noer, H.M., Sollano, J., Sun, H.S., and Xu, D.Z. (2000). Chronic hepatitis B virus infection in Asian countries. J. Gastroenterol. Hepatol. $15,1356-1361$.

Mori, R., Ishiguro, H., Kuwabara, Y., Kimura, M., Mitsui, A., Tomoda, K., Mori, Y., Ogawa, R., Katada, T., Harata, K., and Fujii, Y. (2008). Targeting beta1 integrin restores sensitivity to docetaxel of esophageal squamous cell carcinoma. Oncol. Rep. 20, 1345-1351.

Ng, C.C., Yew, P.Y., Puah, S.M., Krishnan, G., Yap, L.F., Teo, S.H., Lim, P.V., Govindaraju, S., Ratnavelu, K., Sam, C.K., Takahashi, A., Kubo, M., Kamatani, N., Nakamura, Y., and Mushiroda, T. (2009). A genome-wide association study identifies ITGA9 conferring risk of nasopharyngeal carcinoma. J. Hum. Genet. 54, 392-397.

Nyholt, D.R. (2004). A simple correction for multiple testing for single-nucleotide polymorphisms in linkage disequilibrium with each other. Am. J. Hum. Genet. 74, 765-769.

O'Doherty, C., Roos, I.M., Antiguedad, A., Aransay, A.M., Hillert, J., and Vandenbroeck, K. (2007). ITGA4 polymorphisms and susceptibility to multiple sclerosis. $J$. Neuroimmunol. 189, 151-157.

Park, B.L., Kim, Y.J., Cheong, H.S., Lee, S.O., Han, C.S., Yoon, J.H., Park, J.H., Chang, H.S., Park, C.S., Lee, H.S., and Shin, H.D. (2007). HDAC10 promoter polymorphism associated with development of HCC among chronic HBV patients. Biochem. Biophys Res. Commun. 363, 776-781.

Patil, M.A., Lee, S.A., Macias, E., Lam, E.T., Xu, C., Jones, K.D., Ho, C., Rodriguez-Puebla M., and Chen, X. (2009). Role of cyclin D1 as a mediator of c-Met- and beta-catenin-induced hepatocarcinogenesis. Cancer Res. 69, 253-261.

Shin, H.D., Park, B.L., Cheong, H.S., Yoon, J.H., Kim, Y.J., and Lee, H.S. (2007). SPP1 polymorphisms associated with HBV clearance and HCC occurrence. Int. J. Epidemiol. 36, 1001-1008.

Shin, H.D., Park, B.L., Kim, L.H., Jung, J.H., Kim, J.Y., Yoon, J.H., Kim, Y.J., and Lee, H.S. (2003). Interleukin 10 haplotype associated with increased risk of hepatocellular carcinoma. Hum. Mol. Genet. 12, 901-906.

Stephens, M., Smith, N.J., and Donnelly, P. (2001). A new statistical method for haplotype reconstruction from population data. Am. J. Hum. Genet. 68, 978-989.

Streuli, C.H., and Akhtar, N. (2009). Signal co-operation between integrins and other receptor systems. Biochem. J. 418, 491-506. 
Supplementary Table 1. Primer sequences for ITGB1 SNP screening

\begin{tabular}{lll}
\hline & \multicolumn{1}{c}{ Forward } & \multicolumn{1}{c}{ Reverse } \\
\hline Frag-1 & TGATGCAGTTAGATTCCCTGT & AACTACCCCAATTTCCTACCA \\
Frag-2 & TCTTCTTGTGGAGGGACAGA & CACCTGAGGTCAGGATTTG \\
Frag-3 & TGAGATGGAGTCCTGCTCTG & GAATCCATTCGTGCCTTCT \\
Frag-4 & CCGCAGGAGATAATGGAGAT & GCCTGACCATGAAGAACTT \\
Frag-5 & AGGGGATGAATACCCCATTT & CCACATCTGCCTAGTGGCTA \\
Frag-6 & ACATCGGGTTGTTCAGCATA & TGCTGTGGTTGGATCTGAGT \\
Frag-7 & TGCCCTCCAGATGACATAGA & ACAGGGCCACTCTACCATTC \\
Frag-8 & TATCTTTGCATCCTGAAGGC & AGCATCCAGTGACAGAGGAA \\
Frag-9 & CACCTTCCAAAGAACCACT & ATGAATGCGCTTACTCCACA \\
Frag-10 & CAGGATTTGGCTCATTTGTG & GCAATTTCAAGGTTCCTGGT \\
Frag-11 & AGAGCTTCATTCAGTGGGCT & GGGAGTTGAGGCAAAGAGTG \\
Frag-12 & AGAGCTTGTGGGTGACTGG & CTGCTGAAGTGAGTCGTGTG \\
Frag-13 & TTGGCAAAGCATTCAAGTTC & TCCCACATTCAATTCAGACA \\
Frag-14 & TGCTCACCTTGTCCAGAACC & TGGGCTCGCTAAAGTGTGTA \\
Frag-15 & TATCCCATTTCCTTCATGACAC \\
Frag-16 & AAACTGTTCTCTGGCCTCTG & TTGTTGAATATACTTCTTTCATGGC \\
Frag-17 & TTTCCATTGGAGATGAGGTATG & CCCTCAAGCTACCCCTTTC \\
Frag-18 & TGAACCAGTACCTCAGTTGG & GCTTAGGAGAGCCAAGAGGA \\
Frag-19 & AACAATGGAGAGTGCGTCTG & GATTACAGGCACACACCACC \\
Frag-20 & GATGTGTCAGACCTGCCTTG & GGCTCTGCACTGAACACATT \\
Frag-21 & CACACCTGTAATCCCAGCAC & TCAGGCATCGTTCTAAGTGTCT \\
Frag-22 & TTCATTTATTGAAATCTCCTTCA & ACTGGCCACAGTAACCAGAA \\
Frag-23 & TTGATCATGTATCCTGCAACCT & CAGAAACTCTCATCATGCTCATT \\
Frag-24 & AATAGCACCAGATAACGGGG & TTCCCATGGCCTTTGAGAT \\
Frag-26 & TGGTTTACTCATGTGCAGG & AGAGGTGACAGAAAGCACCA \\
Frag-27 & TATAGCGATTGAAAGGCAA & TGAACTACCCACCAACCAGA \\
\hline
\end{tabular}


18 Genomics \& Informatics Vol. 8(1) 9-18, March 2010

Supplementary Table 2. Sequences of amplifying, Taqman probe, and extension primer for ITGB1 SNP genotyping

\begin{tabular}{|c|c|c|c|}
\hline Loci & rs\# & & Probe sequence \\
\hline \multirow[t]{4}{*}{$-401 T>C$} & rs2504001 & Forward & GCATTGAGGTAACTGAGGCATCTG \\
\hline & & Reverse & TCTCCTGCGGCTCCCA \\
\hline & & VIC & CCCAGACCAGCATAT \\
\hline & & FAM & CCAGACCGGCATAT \\
\hline \multirow[t]{4}{*}{$-170 C>A$} & rs 2245840 & Forward & CGCAGTGTAGGTGCAAGGT \\
\hline & & Reverse & CAGACCCTCGCCCATCTC \\
\hline & & VIC & СCTCCCTAGCTGGTTC \\
\hline & & FAM & СССTCCСTATCTGGTTC \\
\hline \multirow[t]{4}{*}{$+5479 A>C$} & rs2256455 & Forward & GATGTGATTTGATGTTTTGCAATTTAATTGCTT \\
\hline & & Reverse & САТTССТTССТGTAАAАATGTCTAAATGACA \\
\hline & & VIC & ACCATTACAATTTTCA \\
\hline & & FAM & ACCATTACACTTTTCA \\
\hline \multirow[t]{4}{*}{$+7377 C>T$} & rs7079624 & Forward & GAGAGCTGAAGACTATCCCATTGAC \\
\hline & & Reverse & TCTGTTCCAAGACTTTTTACATTCTCCAA \\
\hline & & VIC & TCTTTCATTGAGTAAGACAG \\
\hline & & FAM & TCTTTCATTGAATAAGACAG \\
\hline \multirow[t]{4}{*}{$+9685 T>C$} & rs2298141 & Forward & TTTCGATGCCATCATGCAAGTTG \\
\hline & & Reverse & TCCCCTGATAGGAAATGAATGCG \\
\hline & & VIC & СТTАСТССАСАААСТG \\
\hline & & FAM & TTACTCCGCAAACTG \\
\hline \multirow[t]{3}{*}{$+33920 G>T$} & rs2153875 & Forward & AATAGCACCAGATAACGGGG \\
\hline & & Reverse & CAGAAACTCTCATCATGCTCATT \\
\hline & & Extension & ATTTTAGTAGGGTAACTGATAATTTTTCTCACTTTTTTTTTGTT \\
\hline \multirow[t]{4}{*}{$+34701 C>T$} & Novel & Forward & ССTTCACTTTACAAATTCAAGCCTTAGA \\
\hline & & Reverse & TGGCCTGTATGTAAATAGTGCTAAATCAAG \\
\hline & & VIC & TTTTAGCAGAAAATTG \\
\hline & & FAM & TTTTAGCAAAAAATTG \\
\hline
\end{tabular}

